\title{
El Impacto de la Alienación Laboral sobre las Conductas Desviadas en el Trabajo: Un Estudio Exploratorio
}

\section{The Impact of Work Alienation on Workplace Deviance: An Exploratory Study}

\author{
Pablo Zoghbi Manrique de Lara \\ Universidad de Las Palmas de Gran Canaria
}

\author{
Guillermo Caamaño Brito \\ Exalia
}

\begin{abstract}
Resumen. Este estudio examina la alienación laboral (AL) como antecedente del comportamiento desviado en el trabajo (DWB) dirigido contra la organización, los compañeros o los clientes. El modelo analizado se basa en la alienación laboral (AL) de Seeman (1959) y predice que tres dimensiones de AL (powerlessness, meaninglessness y self-estrangement) influyen positivamente sobre las conductas desviadas en el trabajo (DWB). Los datos fueron extraídos de 99 de los 156 (63.5\%) profesores de un distrito de enseñanza media en España. Ecuaciones estructurales (SEM) muestran que, en contra de lo que cabría esperar, las dimensiones estudiadas de AL tienen efectos diferentes, e incluso opuestos, sobre el DWB. Así, el meaninglessness (MLN) y el powerlessness (PLN) mostraron influencias significativas sobre el DWB, pero ésta fue negativa en el caso del powerlessness (PLN). El influjo del self-estrangement (SES) no fue significativo. El artículo también ofrece implicaciones, limitaciones y futuras investigaciones.

Palabras Clave: burnout, conductas desviadas en el trabajo, alienación laboral.
\end{abstract}

\begin{abstract}
This study examines work alienation (WA) as an antecedent of the workplace deviance targeting the organization, co-workers and clients. The model tested is based on the Seeman's (1959) definition of work alienation (WA), and predicts influences of the three WA dimensions of WA (powerlessness, meaninglessness, and self-estrangement) on workplace deviance. Data were collected from 99 of the 156 $(63.5 \%)$ teachers at a district high school in Spain. Structural equation modeling (SEM) results show that, contrary to expectations, WA dimensions exerted different, and even opposite, significant effects on workplace deviance. As such, meaninglessness (MLN) and powerlessness (PLN) showed significant influences on workplace deviance, but in the case of powerlessness (PLN) this association was negative. Self-estrangement (SES) failed in predicting workplace deviance. Implications of the results are discussed, and limitations and future research directions are also offered.

Key Words: burnout, workplace deviance; work alienation.
\end{abstract}

Los comportamientos desviados negativos en el trabajo, Robinson y Bennett (1995) los denominan en la literatura anglosajona como deviant workplace behaviors (DWB) y se producen cuando "de forma voluntaria [los empleados] violan importantes normas organizativas [...] poniendo en peligro el bienestar de la organización, de sus miembros o de ambos a la vez" (p. 556, [énfasis añadido]). Ejemplos de DWB dirigido hacia la organización incluirían el robo, el sabotaje, el retraso doloso al trabajo o poner poco empeño en las tareas exigibles, entre otros. Cuando estas conductas son dirigidas hacia otras personas, las burlas, las agresiones, el acoso y el favoritismo constituirían ejemplos del DWB interpersonal.

Con creciente interés las literaturas sobre psicología del trabajo y dirección de recursos humanos buscan las causas de este "mal comportamiento organizacional".

La correspondencia debe enviarse al primer autor, Universidad de Las Palmas de Gran Canaria, Departamento de Economía y Dirección de Empresas, Campus Universitario Tafira. Edificio de CC. Económicas y Empresariales, Módulo C-2.18, 35017 Las Palmas de Gran Canaria, correo electrónico:pzoghbi@dede.ulpgc.es
Este interés parece justificado. Sólo en términos de costes por pérdida de productividad, por ejemplo, el DWB cuesta anualmente a organizaciones estadounidenses 4.200 millones de dólares (Bensimon, 1994). Otras investigaciones confirman que el $75 \%$ de los empleados han sustraído material de su empresa al menos en una ocasión (Coffin, 2003) y que el 95\% de las organizaciones declaran haber sufrido alguna vez robos por parte de sus empleados (Case, 2000; Robinson, 2008). Desde la perspectiva del DWB interpersonal, también Gruber (1990) constata que un $42 \%$ de las mujeres declaran haber sido acosadas en su trabajo, mientras que Schat y Kelloway (2005) la alta prevalencia de la violencia en el trabajo. En España, el Barómetro europeo del hurto en la distribución, presentado por Checkpoint Systems (Molist, 2005), afirma que las empresas de distribución pierden 1.756 millones de euros al año por causa de hurtos, lo que representa el $1.29 \%$ de las ventas de este sector. Desglosadas, el $52 \%$ de dichas pérdidas son provocadas por hurtos de clientes, mientras que el $25 \%$ corresponden a hurtos de los propios empleados. Ante tal escenario, 
urge encontrar las causas que conducen a los empleados a desviar sus conductas laborales (DWB) en las organizaciones.

La alienación (del latín, alienus: “ajeno", "extraño") es un fenómeno estudiado no sólo por la psicología, sino por la medicina, la sociología, la economía, e incluso la filosofía (Johnson, 1973). Sherpard (1973) define la alienación como la conciencia de discrepancia entre los propios valores y las demandas de la realidad. Fromm (1955) concibió la alienación como un modo de experiencia según la cual una persona se siente ajena o alejada de sí mismo. Horowitz (1966) sugiere que la alienación implica una intensa desconexión o desarraigo de un individuo de las cosas, gente, e ideas del mundo que le rodea. El mundo del trabajo es uno de los contextos en los que los individuos pueden experimentar la alienación, afectando por tanto a los empleados de una organización. Sin embargo esta alienación laboral, que ha sido ampliamente estudiada por las ciencias sociales, tiene una presencia escasa en la literatura organizacional (véase Mottaz, 1981 y Sarros et al., 2002, para una revisión). Es verdad que Sarros et al. (2002) verifican que los estilos de liderazgo insensibles y poco motivadores (frente a los transformacionales) pueden provocar la aparición de la alienación en los supervisados. También Hirschfeld y Feild (2000) encuentran que el compromiso afectivo está fuertemente asociado a la alienación. Sin embargo, desafortunadamente, tales estudios sobre alienación laboral no examinan sus potenciales efectos sobre el comportamiento organizacional $\mathrm{y}$, hasta donde nosotros sabemos, no existen estudios previos sobre esa relación en el contexto organizacional.

La alienación laboral (o simplemente alienación o $\mathrm{AL}$, de aquí en adelante) tiene su origen en los escritos del filósofo alemán Karl Marx (1844), el cual la entendía como el resultado de una contradicción entre el trabajo de un empleado y su propia naturaleza humana. Arranca en la filosofía idealista alemana que, a su vez, entiende la alienación como el alejamiento del sujeto con respecto de sí mismo, perdiendo así su autenticidad. Más concretamente, Marx (1844) dibujó la AL como un conjunto de disfunciones generadas en el individuo por la mencionada fricción de su condición humana contra la estructura de la sociedad capitalista de la época. El individuo, aseveraba Marx (1844), ante un capitalismo deshumanizado, perdía el control sobre el producto y el propio proceso de producción, asî́ como su capacidad de expresarse en el trabajo. Como resultado, el trabajador no encuentra significado a sus tareas laborales pudiendo presentar síntomas de desorientación, incapacidad o impotencia, así como de futilidad y aislamiento. Más recientemente se han establecidos posibles lazos entre la AL y el síndrome del "burnout" (Maslach y Jackson, 1980, 1984), aunque dicho síndrome tiene su origen en múltiples causas y sus consecuencias no son siempre conductuales.

El presente trabajo sugiere que entre los menciona- dos efectos indeseables que la alienación puede provocar en los empleados está el que se comporten desviada o inapropiadamente (DWB). En este sentido, ya Akroyd y Thompson (2000) consideraron al DWB como una vía a través de la cual el trabajador alienado intenta recuperar el dominio y control perdidos sobre el proceso productivo de su organización. Sin embargo, la literatura organizacional no proporciona ningún apoyo empírico a tal aserto, así como a cualquier posible vinculación entre la AL y DWB. Por lo tanto, hasta donde nosotros conocemos, la alienación laboral (AL) como antecedente de los comportamientos desviados laborales (DWB) no ha sido estudiada empíricamente en el seno de las organizaciones. Cubrir este vacío en la literatura constituye el objetivo de este trabajo.

\section{Marco teórico}

Un importante grupo de terminologías y conceptos circundan la desviación de conductas en el trabajo. Así, estas acciones son definidas como comportamientos anti-sociales (Robinson y O'Leary-Kelly, 1998), como "misbehavior" organizacional (Vardi y Wiener, 1996), conductas de incumplimiento (Puffer, 1987), desviación en el lugar de trabajo (Robinson y Greenberg, 1998) o conductas laborales disfuncionales (Griffin, O’Leary-Kelly y Collins, 1998), por nombrar sólo algunas. Sus definiciones también difieren en matices. Así, Gruys y Sackett (2003) las denominan conductas contraproductivas en el trabajo (CWB) y las definen como "cualquier comportamiento intencionado, por parte de un miembro organizacional, que sea visto por la organización como contrario a sus legítimos intereses" (p. 30). Robinson y Bennett (1995), en su definición del DWB anteriormente vista, resaltan también su necesaria intencionalidad, esto es, "voluntariedad", añadiendo que el empleado "[incumple] importantes normas organizacionales" lo que daña a otras personas (DWB-I) y a la propia organización como un todo (DWB-O) (p. 556, [énfasis añadido]; véase también Bennett y Robinson, 2000).

Como anteriormente hicimos constar, los orígenes de la alienación laboral (AL) parten de los escritos de Karl Marx. Según Marx (1844) la alienación incluía cuatro elementos. Los dos primeros son el (a) producto y el (b) proceso de producción, que pertenecerían a los capitalistas y que generalmente lo venden para lucrarse. Con ello, los trabajadores no perciben la real utilidad de su actividad productiva, diluyéndose en largas cadenas de producción a cambio de un simple salario de supervivencia. También Marx (1844) argumenta que, en el capitalismo, los trabajadores están alienados de otros elementos como (c) sus compañeros de trabajo y (d) su propio potencial humano. En el primero, dado que el capitalismo destruiría la cooperación natural de los trabajadores y los aísla, también les convertiría en víctimas de una sensación profunda de soledad. 
En efecto, el capitalista que habitualmente haría competir a los trabajadores entre sí para aumentar su rendimiento, lo que no pocas veces podría ser causa de desconexión y hostilidad entre ellos. También, como Marx (1844) señaló, los trabajadores pudieran están alienados (d) de su propio potencial humano. En efecto, según Marx (1844), los individuos cada vez se realizarían menos como seres humanos, vaticinando que quedarían finalmente reducidos a un papel de "bestias de carga" o "máquinas inhumanas". Su conciencia se entumecería y, como una masa de personas incapaces de expresar sus capacidades nobles, se convertían en trabajadores alienados.

Seeman (1959) proporcionó la interpretación más contemporánea de la alienación, constituyéndose en referente indiscutible de la mayoría de las investigaciones desarrolladas sobre la AL en las ciencias sociales (p.ej., Blauner, 1964; Dean, 1961; Seeman, 1967; Shepard, 1977). Seeman (1959) desgrana el concepto marxista de alienación en cinco dimensiones: powerlessness, meaninglessness, normlessness, isolation y self-estrangement. Así, powerlessness alude a los sentimientos de un trabajador de ausencia de control o impotencia sobre el desempeño de su trabajo diario, según éste está formalmente dispuesto. El sentimiento meaninglessness (falta de significado) estaría presente en el trabajador que ve que su labor genera una escasa influencia sobre el proceso de producción de la organización. Otra dimensión de alienación sería la normlessness o la ausencia de normas, según la cual los trabajadores sentirían que las normas son escasas, confusas o que han perdido su valor. También, Seeman (1959) referenció la isolation, es decir, el sentimiento de aislamiento o de ausencia de identificación del trabajador con su organización. Por último, la quinta dimensión de Seeman (1959) es el self-estrangement. Este sentimiento estaría presente entre los trabajadores de una organización cuando no se sienten realizados con la labor que desempeñan en la misma. Shepard (1977) vió esta última dimensión como aquélla en donde los trabajadores sienten el trabajo como un medio para alcanzar otros fines, pero nunca los de autorrealización personal. El trabajo es algo, pues, meramente instrumental.

La reformulación de Seeman (1959) de la alienación a nivel individual culmina en Mottaz (1981) cuando éste presenta a la comunidad científica su constructo multidimensional que contempla tres facetas de la alienación, (1) powerlessness (PLN), (2) meaninglessness (MLN) y (3) self-estrangement (SES), con su correspondiente escala de medición. Cabe señalar que la dimensiones de isolation o aislamiento y de normlessness o ausencia de normas propuestas por Seeman (1959), no están contempladas por Mottaz (1981). Con respecto a la primera, Mottaz (1981) lo justifica en base a la literatura que -según él- en general no considera que la isolation se ajuste bien con las otras tres dimensiones de la AL, ni conceptualmente (Blauner,
1964; Kirsch y Lengermann, 1971) ni empíricamente (p.ej., Kohn, 1976). Así, si bien PLN, MLN y SES se refieren al trabajo en sí mismo, Mottaz (1981) argumenta que la isolation se refiere más bien a aspectos relacionados con la organización y los compañeros de trabajo. También, Mottaz (1981) apunta a estudios previos según los cuales la dimensión normlessness debe ser también obviada. En esta ocasión apoyándose en el estudio previo de Blauner (1964), Mottaz (1981) no considera que sea una dimensión diferente ni vinculada a antecedentes laborales sino, como en el caso de la anomia (del Griego, an-: ausencia, y -nomos: ley, norma) de Emile Durkeim (1893, 1897), más bien relacionada con causas sociológicas.

En el presente artículo, es en este constructo tridimensional de alienación de Mottaz (1981), en donde se asienta nuestro trabajo. Con respecto a las variables dependientes, en la última década, un sinfín de estudios ha escogido el constructo DWB de Robinson y Bennett (1995) como una medida indiscutible de la conducta desviada en las organizaciones. Este estudio también.

\section{Hipótesis}

Las teorías de intercambio social (Blau, 1964; Gouldner, 1960) parecen constituir un paradigma adeudado para justificar nuestras predicciones. Según estas teorías, la vida social es concebida como un constante fluir de intercambios, en donde los individuos se saben necesitados de los demás para perpetuar los beneficios que reciben (Blau, 1964; Gouldner, 1960). Un marco habitual de intercambios es el constituido por las relaciones entre la organización y sus miembros, en donde cada parte sopesa su comportamiento con el de los demás - en función de los beneficios que puede recibir - para perpetuar una dinámica beneficiosa de intercambios para ambas partes. Foa y Foa (1975) resaltan los intercambios no materiales (respeto, estatus, beneficios sociales, etc.) frente a los materiales, más habituales, como el dinero y otros. En el mundo laboral, cada parte (empleados, compañeros, clientes y organización) es correspondida por los beneficios que suministra a la otra. Por tanto, los protagonistas que componen el contexto social del empleado alienado (sus compañeros, clientes y su organización) justificarían las tres respuestas selectivas que reflejan nuestras hipótesis: DWB contra la organización (DWB-O), contra los compañeros (DWB-I-C) y contra los clientes (o alumnos en nuestra muestra, como veremos), DWB-I-A. La primera refleja una respuesta 'contra' la organización (DWB-O), las dos últimas contra individuos o interpersonales (DWB-I).

De acuerdo con la mecánica de intercambios sociales, Blau (1964) sugiere que la conducta del individuo busca radicalmente acciones que le reporten recompensas. Cuando éstas no se dan, sus respuestas cesan. 
En base a tal principio de quid pro quo, cabría esperar que el empleado alienado señale a su organización como responsable de su alienación y la culpabilice de ese sentimiento de frustración personal. Como resultado, parece razonable pensar que los empleados podrían vengarse por su alienación mostrando DWB contra su entidad. Robinson y Bennett (1995) apoyan tal argumentación cuando sugieren que si la culpa es atribuida a la organización como un todo, es "más probable que determine conductas del empleado dirigidas a dañar directamente a la organización [DWB-O] y que las variables individuales [si la es culpa atribuida a los demás] es más probable que desencadenen formas de desviación interpersonal [DWB-I-C y DWB-I-A]" (p. 567, [énfasis añadido]). Por tanto, parece razonable pensar (véase la Figura 1) que mientras el empleado más se perciba alienado de su organización, con más frecuencia e intensidad replicará específicamente 'contra' ella (DWBO). Por ello, nuestras primeras hipótesis serían:

Hipótesis 1a: Los empleados que presenten un mayor sentimiento de falta de control sobre el desempeño su trabajo (PNL) mostrarán más conductas desviadas contra su organización (DWBO).

Hipótesis 1b: Los empleados que presenten un mayor sentimiento de escasez de contribución al proceso productivo de su organización (MLN) mostrarán más conductas desviadas contra su organización (DWBO).

Hipótesis 1c: Los empleados que presenten un mayor sentimiento de falta de realización con la tarea que realizan en su organización (SES) mostrarán más conductas desviadas contra su organización (DWBO).

Los grupos de trabajo en una organización constituyen uno de los referentes más sólidos e influyentes del soporte social (y desarraigo, por tanto) de un empleado alienado. Sus compañeros en dichos grupos de trabajo pudieran constituirse en blanco de sus comportamientos desviados en la medida en que dicho empleado se perciba alienado de ellos, es decir, no dignos de confianza y simples objetos de una relación profesional superficial e inauténtica. De nuevo, basándonos en los principios del intercambio social, el empleado alienado podría corresponder a la 'fuente' de tales negativas percepciones (los compañeros), incrementando su DWBIC. Por consiguiente, nuestras siguientes hipótesis son:

Hipótesis 2a: Los empleados que presenten un mayor sentimiento de falta de control sobre el desempeño su trabajo (PLN) mostrarán más conductas desviadas interpersonales dirigidas contra sus compañeros de trabajo (DWBIC).

Hipótesis 2b: Los empleados que presenten un mayor sentimiento de escasez de contribución al proceso productivo de su organización (MLN) mostrarán más conductas desviadas interpersonales dirigidas contra sus compañeros de trabajo (DWBIC).

Hipótesis 2c: Los empleados que presenten un mayor sentimiento de falta de realización con la tarea que realizan en su centro (SES) mostrarán más conductas desviadas contra sus compañeros de trabajo (DWBIC).

Figura 1. Modelo Propuesto que predice la Alienación Laboral (AL) como causa de la Conductas Desviadas en el Trabajo (DWB)

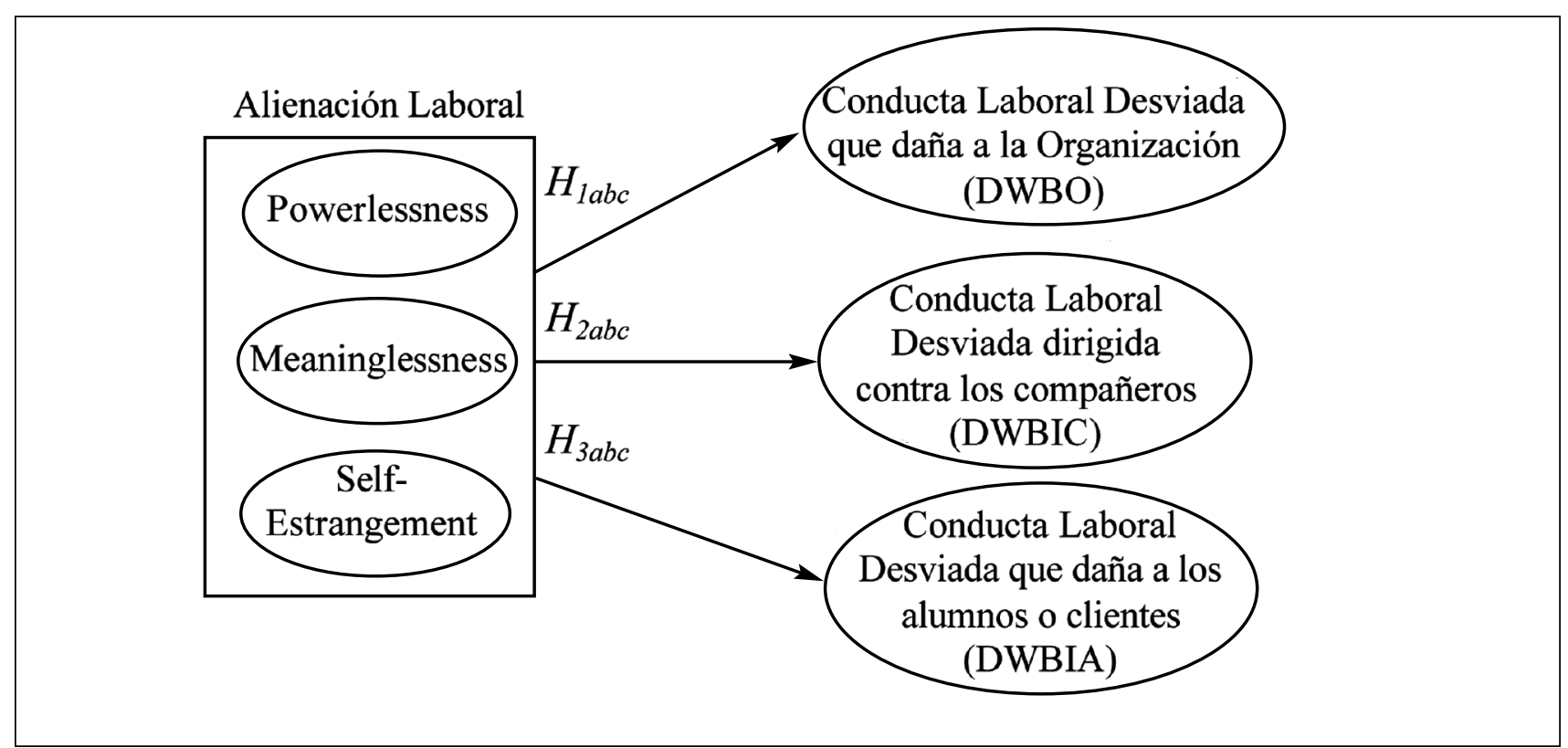


Según las normas de reciprocidad, en los intercambios sociales la 'inversión causal' es posible (Gouldner, 1960). En un estudio previo, Caruana et al. (2001) constatan que los empleados anómicos son menos sensibles a apercibirse de conductas disfuncionales e injustas de sus clientes. Ello significaría que, de la misma manera que, según Caruana et al. (2001), la mayor anomia de los trabajadores pudiera hacerles percibir con mayor indiferencia las conductas disfuncionales e injustas de sus clientes, apelando a la 'inversión causal' aludida, los clientes, con sus conductas, pudieran análogamente constituirse en un factor propiciatorio de la alienación de los empleados con los que interactúan. Por tanto, en base a tal argumentación, los clientes análogamente pudieran erigirse también en 'fuente' o 'causa' de alienación y, por tanto, en 'blanco' contra los que los empleados replicarían incrementando su DWBIA. Por consiguiente, nuestras últimas hipótesis serían:

Hipótesis 3a: Los empleados que presenten un mayor sentimiento de falta de control sobre el desempeño su trabajo (PLN) mostrarán más conductas desviadas interpersonales contra sus clientes (alumnos) (DWBIA).

Hipótesis 3b: Los empleados que presenten un mayor sentimiento de escasez de contribución al proceso productivo de su organización (MLN) mostrarán más conductas desviadas interpersonales contra sus clientes (alumnos) (DWBIA).

Hipótesis 3c: Los empleados que presenten un mayor sentimiento de falta de realización con la tarea que realizan en su organización (SES) mostrarán más conductas desviadas contra sus clientes (alumnos) (DWBIA).

\section{Método}

\section{Ámbito del Estudio y Trabajo de Campo}

Los cuestionarios fueron distribuidos personalmente a todos los profesores de enseñanza de secundaria de un distrito de Gran Canaria (España). El ratio de respuestas fue del 63.5\% (99 de 156), finalizando el trabajo de campo el 20 Febrero de 2006. La muestra estaba compuesta por un $53 \%$ de varones y un $47 \%$ de mujeres y, mientras que el 3\% tenía 30 años o menos, el $55 \%$ era mayor de 40 . En lo que respecta a la antigüedad, el $36 \%$ de los respondientes tenía menos de 10 años, el $50 \%$ tenía entre 10 y 20 años de experiencia en dicha organización y el $14 \%$ tenía 20 ó más. Además, las estructuras descriptivas de la muestra y población son similares. En efecto, en el universo el $53 \%$ de los encuestados tenía entre 10 y 20 años de antigüedad, mientras que el $14 \%$ tenía más de 20 . Por edad, el 3\% tenía 30 años o menos y el $66 \%$ era mayor de 40 años. Por género, el citado universo se distribuía al 50\%.

\section{Medición de Variables}

Las respuestas del estudio fueron registradas sobre una escala tipo Likert de 7 puntos, oscilando desde (1) 'Máximo desacuerdo' a (7) 'Máximo acuerdo' (en el caso de DWB de (1) 'Nunca' a (7) 'Constantemente'). Todos los ítems utilizados en este estudio pueden apreciarse en el Apéndice I. La fiabilidad fue establecida a través del alfa de Cronbach, cuyos valores se muestran en la diagonal principal de la tabla de correlaciones (Tabla 1).

Alienación laboral. Usamos una escala tipo Likert de 7 puntos, con los 21 ítems de Mottaz (1981), que incluyen las dimensiones de powerlessness, meaninglessness, and self-estrangement (7 ítems cada una). Algunos ítems que estaban formulados inversamente fueron recodificados. Antes del cálculo de las alfas eliminamos aquellos ítems cuya carga resultó inferior a .60 , que finalmente resultaron ser $0.82,0.81,0.78$ respectivamente. Como se puede apreciar en el Apéndice I, el número de ítems finalmente elegidos fue de 3 para powerlessness, 4 para meaninglessness y 3 para selfestrangement.

Comportamientos DWB. La escala tipo Likert de 7 puntos de 10 ítems utilizada para calibrar el DWB fue una versión más reducida de la 18 ítems propuesta por Bennett y Robinson (2000). En efecto, ciertos ítems relacionados con conductas xenófobas o religiosamente discriminatorias, entre otras, consideramos no procedían en dicho colectivo estudiado. Por ello, ocho fueron desechados. Se añadieron 3 ítems más para medir el DWBIA dirigido hacia al alumno. Estos últimos fueron diseñados por los autores siguiendo el mismo patrón del DWBI. Los 13 ítems resultantes tenían, pues, pretensiones de tridimensionalidad (DWBO, DWBIC, DWBIA).

Para comprobar si los constructos/dimensiones propuestos eran realmente distintos llevamos a cabo un análisis factorial confirmatorio (AFC) utilizando modelos estructurales (SEM). Los datos los recoge el Apéndice I final. A continuación, los ítems no desechados (ver Apéndice I) fueron promediados para calcular su media, e incorporados a la Tabla 1 de correlaciones. Para buscar apoyo a las hipótesis diseñamos un modelo SEM donde introdujimos todas nuestras variables. Los resultados pueden apreciarse en la Figura 2. En este último caso, usamos las variables promediadas según su media acumulada, como si fueran variables observadas, dada la limitación del SEM tal y como sugiere Aquino et al. (1999) y Bentler y Chou (1987) para operar con modelos con un ratio bajo de indicadores/observaciones, como es nuestro caso, y por la dificultad apuntada por Jöreskog y Sörbom (1986) y Williams y Hazer (1986) para conseguir ajustes acep- 
tables en estos casos. Los resultados obtenidos son presentados en el apartado siguiente.

Finalmente, dado que la AL y DWB son constructos únicos si bien multidimensionales, tanto para el caso de la AL como para el del DWB creímos conveniente el llevar a cabo un análisis SEM que indague en la posible presencia de un factor general de orden superior correspondiente a AL y DWB. Las altas correlaciones entre las dimensiones de cada uno de estos constructos, tal y como que puede verse en la Tabla 1, también sugieren dicha presencia. Por todo ello, se acometió el cálculo de dichos factores latentes de orden superior que incluyen todas las dimensiones en ambos constructos, lo que nos permitirá un análisis de los efectos globales de la AL sobre el DWB.

\section{Resultados}

El AFC que recoge el Apéndice I confirma que nuestras variables están relacionadas pero son diferentes. Aunque la chi-cuadrado resultó ser significativa, el ajuste aportado por el cálculo de los índices del modelo SEM fue suficiente $(\mathrm{Cmin}=297.131, d f=215$, $p<.001, \mathrm{Cmin} / d f=1.382, \mathrm{CFI}=.91, \mathrm{TLI}=.88, \mathrm{IFI}=.91$, $\mathrm{NFI}=.75$, RMSEA $=.062$, PNFI $=.58$ ). Antes de su cálculo, eliminamos aquellos ítems cuya carga resultó inferior a 60 . nación incita al DWB, no parece recibir un inicial sostén.

Como ya anunciamos previamente, para analizar las relaciones entre las variables diseñamos un modelo de ecuaciones estructurales (SEM) introduciendo las variables promediadas. El modelo ofreció un ajuste excelente y, por ello, podemos aceptar sus relaciones. En efecto, la Chi-cuadrado resultó ser apenas significativa $\left(\chi^{2}[3,99]=8.387 ; p=.039\right)$, el GFI fue .97; el TLI fue .91; el CFI fue .94; el NFI = .92; y el RMR fue .04. A continuación, procedimos a analizar cada una de las relaciones obtenidas (Figura 2). Así, los resultados parecen respaldar y por tanto nos permiten aceptar las Hipótesis $2 \mathrm{a}$ y $2 \mathrm{~b}$, ya que el MLN incrementó significativamente el DWBO $(B=.22 ; p<.05)$ y el DWBIC $(B=.27 ; p<.01)$. Con sorpresa, la relación de PLN con el DWBO $(B=-.21 ; p<.05)$, el DWBIC $(B=-.29$; $p<.01)$ y el DWBIA $(B=-.29 ; p<.01)$ resultó significativa, pero en dirección opuesta a la esperada (Figura 2), por lo que las Hipótesis 1a, 1b y 1c no reciben apoyo. Las demás relaciones no mostraron significación, por lo que rechazamos las Hipótesis 3a, 3b, 2c y $3 \mathrm{c}$.

Finalmente, puesto que parece existen dos factores generales de orden superior se examinó la relación general entre AL y DWB, además de las relaciones entre las subdimensiones de cada uno de los constructos, cuyos resultados ya se han comentado. Aunque el

Tabla 1. Medias, Desviaciones Estándar, Correlaciones y Fiabilidades

\begin{tabular}{|c|c|c|c|c|c|c|c|c|}
\hline Variables & $M$ & $D E$ & 1 & 2 & 3 & 4 & 5 & 6 \\
\hline 1. Powerlessness (PLN) & 3.13 & 1.14 & $(0.820)$ & & & & & \\
\hline 2. Meaninglessness (MLN) & 3.04 & 0.99 & $0.557 * * *$ & $(0.810)$ & & & & \\
\hline 3. Self-Estrangement (SES) & 3.19 & 1.20 & $0.500 * * *$ & $0.532 * * *$ & $(0.784)$ & & & \\
\hline 4. DWBO (organización) & 2.02 & 0.73 & -0.066 & 0.125 & 0.094 & $(0.815)$ & & \\
\hline 5. DWBIC (compañeros) & 1.90 & 0.83 & -0.147 & 0.092 & 0.030 & $0.485 * * *$ & $(0.742)$ & \\
\hline 6. DWBIA (clientes o alumnos) & 2.19 & 0.97 & $-0.234 * *$ & -0.088 & -0.078 & $0.430 * * *$ & $0.436 * * *$ & $(0.732)$ \\
\hline
\end{tabular}

Nota. En la diagonal principal se ofrecen los coeficientes Alfa de Cronbach. Las alfas fueron calculadas una vez los ítems con una carga inferior a .60 fueron descartados, según el AFC conducido y mostrado en el Apéndice I.

$N=99 . * p<0.1 ; * * p<0.05 ; * * * p<0.01$.

La Tabla 1 muestra los estadísticos descriptivos, las correlaciones $(r)$ y las fiabilidades de todas las variables del estudio. Los resultados del análisis muestran poco sustento a las hipótesis propuestas. En general, sería de esperar correlaciones positivas entre AL y comportamientos desviados en el trabajo (DWB) en todos los ámbitos (contra a la organización, los compañeros y los clientes o alumnos). Sin embargo, ninguna de las dimensiones de la alienación estudiadas parece correlacionarse positivamente con las conductas desviadas contra la organización (DWBO), compañeros (DWBIC) o alumnos (DWIA). Más bien al contrario, la dimensión PLN parece reducir la aparición de conductas desviadas DWBIA $(r=-.234 ; p<.05)$. Por tanto, la tesis general de nuestro estudio de que la alie- modelo se ajusta mejor que el de orden inferior antes analizado (véase Apéndice II), los resultados de las dos variables latentes globales muestran un influjo no significativo $(B=-.04 ; p$ ns) de la AL sobre el DWB tal y como se muestra en el Apéndice II. En general, la AL no parece mostrar un efecto sobre el DWB.

\section{Discusión}

El objetivo de esta investigación fue analizar el impacto que la AL tiene sobre el DWB, una relación que, a pesar de la profusión de estudios sobre la AL, ha sido escasamente estudiada sobre todo en el ámbito de las organizaciones. El presente análisis arroja resulta- 
dos que, aunque inesperados, pueden ofrecer importantes implicaciones y permitirle a las direcciones organizativas concretar un abanico de opciones a la hora de combatir el DWB. Así, dicha nueva perspectiva que la $\mathrm{AL}$ ofrece en la lucha contra tales conductas desviadas pudiera permitir mejoras en el rendimiento organizacional. Tales propósitos, conjugados con los distintos matices que ofrecen nuestros resultados, nos proponen conclusiones e implicaciones que pasamos a desarrollar y comentar en las líneas que siguen.
(MLN). Los alumnos, por contra, parecen permanecer ajenos a tal estructura organizativa alienante, puesto que el DW-I-A no fue afectado por el MLN $(B=.13$; $p$ n.s.). Probablemente, los empleados (profesores) perciben mayormente a los alumnos/clientes como una presencia circunstancial o, incluso, como víctimas colaterales de ese trabajo falto de significado (MLN).

Recordemos que la situación de PLN refiere un entorno de trabajo donde el individuo no tiene control sobre la forma en que lleva a cabo su trabajo y donde

Figura 2. Modelo Aceptado de la Alienación, en sus tres Dimensiones y en las tres Dimensiones de las Conductas Desviadas en el Trabajo (DWB)

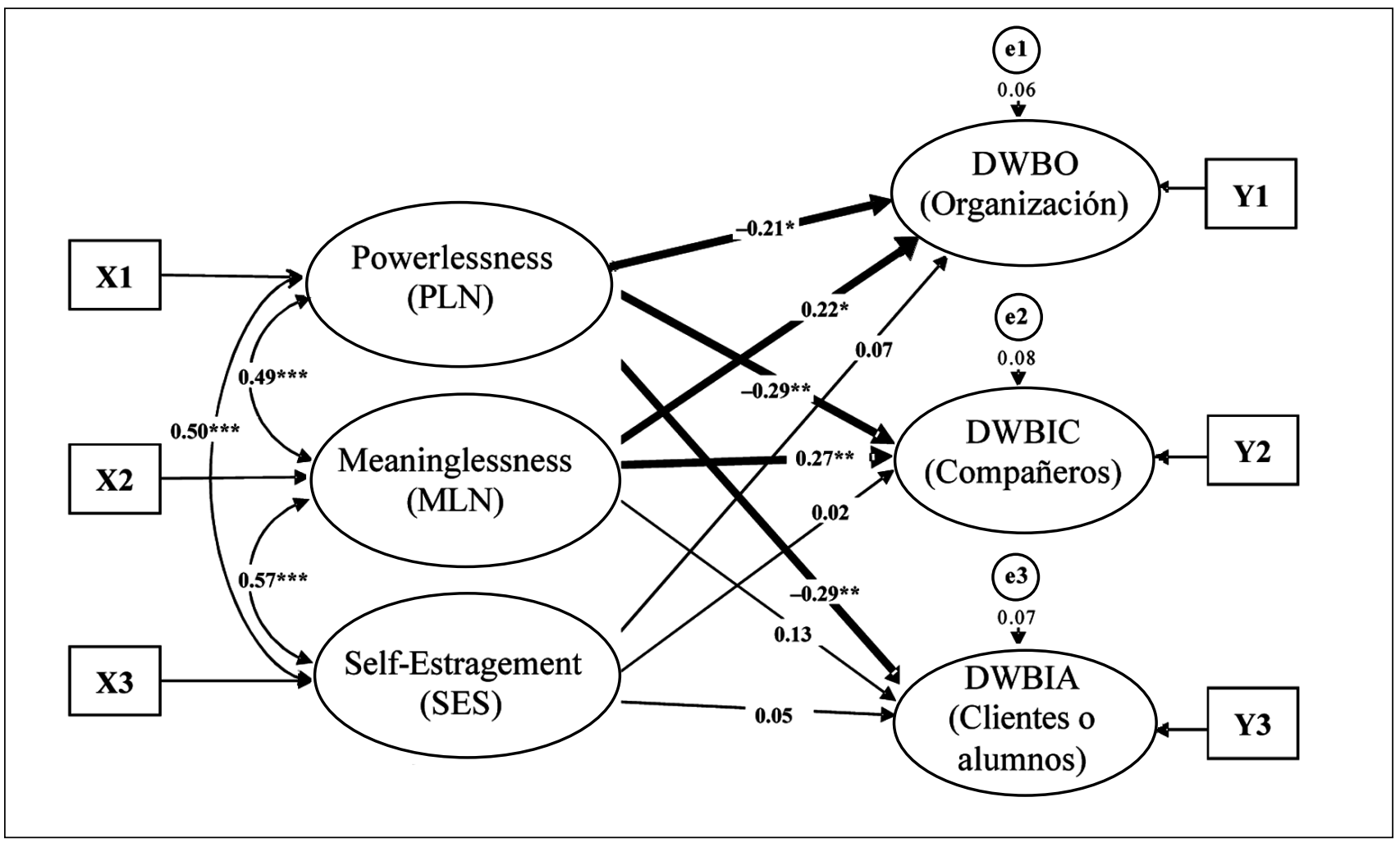

$N=99 . * p<0.05 ; * * p<0.01$.

Nota: Las relaciones significativas se ofrecen en trazo grueso.

En primer lugar, los datos extraídos del modelo SEM apoyan una relación causal entre la dimensión MLN y, tanto el DWBO $(B=.22 ; p<.05)$ como el DWBIC $(B=.27 ; p<.01)$. Tales resultados están dentro de lo a priori esperado, esto es, los sujetos muestran una mayor incidencia de DWB como respuesta a un trabajo sin sentido. Con lógica, al menos entre los empleados de nuestra muestra, tales conductas se desarrollarían contra la organización como ente responsable de determinar esas condiciones y contenido del trabajo causantes de MLN, pero también contra los compañeros (DWB-IC) como un elemento percibido más dentro de esas condiciones de trabajo. Por ello, tanto organización como compañeros parecen formar condiciones laborales percibidas como carentes de sentido su capacidad de decisión está muy restringida o anulada. Los resultados aquí muestran una relación inversa muy significativa entre el factor PLN y las tres variantes de DWB, y más relevante en el caso de las conductas desviadas dirigidas contra los compañeros $(B=$ $-.29 ; p<.01)$ y clientes/alumnos $(B=-.29 ; p<.01)$. Ello contradice nuestra tesis de existencia de influencia positiva de esa sensación de impotencia o de falta de control en el trabajo (PLN) sobre conductas desviadas en contra de la organización (DWB-O), compañeros (DWB-I-C) y clientes/alumnos (DWB-I-A). ¿Cómo explicar este sorprendente resultado?

Una primera razón pudiera estar en las características concretas de nuestra muestra, es decir, los profesores pudieran achacar su descontento con la disposición 
actual de su trabajo no a los responsables del centro sino a políticas públicas de altas instancias, a las que su centro es ajeno. Como resultado, su disminución de DWB (en las tres dimensiones) pudiera denotar una forma de "compensar" su PLN a través de buscar en su propio centro, compañeros y alumnos una válvula de escape, un lugar afectivamente cálido, que contrarreste su PLN. En efecto, ante la falta de control sobre el trabajo desempeñado, el trabajador (profesor en este caso) no vería a su centro, compañeros y clientes/ alumnos como causantes de su sentimiento de AL sino incluso como víctimas de un sistema de trabajo o una supra-organización que también los aliena a ellos. Ante esto, el trabajador mostraría empatía con ellos reduciendo sus conductas desviadas. Congruentemente con Simpson (1999) y Korman et al. (1981), que subrayaron el contacto interpersonal como un elemento que contrarresta la $\mathrm{AL}$, en nuestro estudio esta relación negativa fue más relevante en el caso de conductas desviadas interpersonales, es decir, dirigidas contra compañeros $(B=-.29 ; p<.01)$ y clientes/alumnos $(B=-.29 ; p<.01)$. Las llamémosle "impersonales", o contra la organización, fueron algo menos significativas $(B=-.21 ; p<.05)$.

En segundo lugar, una segunda explicación podría provenir de la posible existencia de un entorno de trabajo percibido como provocador de PLN pero que, a la sazón, fuera "confortable". En efecto, ante el PLN el individuo busca (y puede conseguir) mantenerse dentro de una "confort-zone" que le permite minimizar los riesgos en que se asumen en el trabajo diario. Aunque este argumento contradice la abundante literatura empresarial que, enarbolando la bandera del empowerment, durante los años 80, asociaría al PLN con menor rendimiento y satisfacción del empleado, no nos olvidemos que la disminución del DWB por el PLN no la interpretamos en clave positiva. En efecto, subrayamos que esta relación inversa a la esperada es indeseable y simplemente podría reflejar los pasos que los empleados darían en el centro estudiado para "intentar" encontrar en dicho centro un lugar "cómodo" ante una PLN inevitable. En otras palabras, ¿para qué dañar al contexto de trabajo que puede hacerme más grata mi estancia laboral?

Sin embargo, ¿no debería esgrimirse esta misma argumentación con respecto a MLN? Creemos, en este sentido, que la dimensión MLN tiene unas connotaciones diferentes al PLN. MLN supone una sensación de falta de significado por parte del trabajador que, a diferencia de PLN, difícilmente se puede enfocar desde una óptica positiva en alusión a la "confort-zone" apuntada. Dicho de otra forma, un trabajador que no tiene una sensación de control sobre su trabajo (PLN) pero que le encuentra algún sentido (MLN), aceptaríamos que pudiera acomodarse en un cierto estatus mínimamente satisfactorio; sin embargo, no creemos que tal afirmación pudiera hacerse a la inversa. En este sentido, podríamos decir que la dimensión MLN es "más alienante" que la PLN, pues daña no sólo el cómo sino el porqué más esencial del significado del trabajo. Además, creemos que el MLN y PLN también difieren en la atribución de culpa que alientan en el profesor. Así, el MLN pudiera verse más probablemente como una responsabilidad a centro, al contrario que en el caso del PLN. Ello explicaría el porqué MLN alienta DWB, al contrario que el PLN. Congruente con este razonamiento, el trabajador (profesor en este caso) falto de sentido en su trabajo desempeñado (MLN) no ve a los clientes/alumnos como causantes de su sentimiento de MLN y, por tanto, los mantiene al margen de sus conductas desviadas $(B=.13 ; p$ n.s. $)$.

Cabe también destacar que los sentimientos de SES perecen ser vistos como ajenos o escasamente de responsabilidad del centro $(B=.07 ; p$ n.s. $)$, compañeros $(B=.02 ; p$ n.s. $)$ o alumnos/clientes $(B=.05 ; p$ n.s. $)$. Desde esta perspectiva, el empleado/profesor parece tener muy interiorizada una visión instrumental de su trabajo, suficiente al menos para no culpar o desahogarse en ninguno de los tres estamentos de DWB propuestos. Ya Rosner y Putterman (1991) sugirieron que la educación aumenta la capacidad y los recursos del individuo para obtener la satisfacción del trabajo, lo que explicaría que la batalla que los profesores libran contra la SES pueda ser más personal o privada y, por tanto, ajena posibles atribuciones de culpa externas a ellos mismos.

Por último, quizá porque en contra de lo que cabría esperar las dimensiones estudiadas de AL tienen efectos diferentes, e incluso opuestos, sobre el DWB, la relación testada sobre la base de los factores latentes globales de orden superior entre la AL y el DWB no resultó ser significativa.

\section{Implicaciones Prácticas}

Quizá principalmente por su impacto sobre la imagen organizativa, sobre todo desde principios de los 80 , las circunstancias en las que se desarrolla la actividad laboral han ido adquiriendo cada vez mayor importancia. En efecto, la seguridad e higiene en el trabajo, las políticas corporativas para garantizar la igualdad de oportunidades o la detección de conductas abusivas dentro de las organizaciones gozan de amplias implicaciones legales y económicas. Sin embargo, a pesar de la abundante literatura sobre AL y del significativo impacto que sobre la productividad y la rentabilidad de las organizaciones tiene el DWB, los esfuerzos de los profesionales de dirección de Recursos Humanos para detectar y mitigar situaciones, métodos o sistemas de trabajo disfuncionales, han repetidamente obviado la óptica que aporta el impacto de la AL sobre el DWB.

Por ello, el mejor conocimiento de las relaciones entre AL y DWB puede ayudar a los profesionales de los recursos humanos y de la gestión organizativa, en general, a seleccionar mejores herramientas y acciones destinadas a minimizar la ocurrencia de DWB y sus efectos 
nocivos sobre la organización. Así, los resultados de nuestro estudio darían soporte a la utilización de estrategias de comunicación dirigidas a aumentar el conocimiento de los trabajadores acerca de la importancia del trabajo que desempeñan y de cómo éste encaja en el resto de actividades de la empresa con el fin de evitar sentimientos de MLN. Y esto no sólo por el ya consabido efecto positivo que tales acciones tendrían sobre el nivel de motivación de los trabajadores, sino también por la reducción de conductas nocivas (DWB) que cabría esperar contra la organización y compañeros de trabajo.

Por el contrario, los resultados de nuestro análisis parecen indicar que los esfuerzos destinados a la erradicación de DWB centrados en evitar el sentimiento de PLN, cuando menos, no serían eficaces. Sin embargo, en nuestra opinión, ello no implicaría que una política de mayor empowerment pudiese llegar a desencadenar conductas DWB. Por contra, aunque no esté apoyado por los resultados del estudio, una política encaminada a erradicar sentimientos de PLN (siempre que su responsabilidad fuese claramente asumida por estamentos próximos al empleado) debería también actuar positivamente, disuadiendo el DWB.

\section{Limitaciones del Estudio y Futuras Investigaciones}

Conviene advertir de ciertas limitaciones que cree- mos adolece nuestra investigación. En primer lugar, la institución docente estudiada reúne ciertas condiciones laborales inherentes a las peculiaridades del trabajador público que limitarían la generalización de los resultados especialmente a colectivos privados. En segundo lugar, el estudio ha seguido un método transversal (cross-sectional) lo que implica que todos los datos tienen la misma fuente (auto-informe) y fueron registrados en un único periodo de tiempo. Ello puede limitar la significación de nuestros resultados. En último lugar, la utilización de constructos diseñados/adaptados por los autores, como el DWB-I-A, mientras no adquieran un contraste pleno sobre su validez, creemos deben anotarse también como limitaciones del presente trabajo.

Finalmente, en lo relativo a futuras investigaciones, sería de interés el profundizar en el análisis de la relación entre el sentimiento de PLN y su antónimo, el empowerment, así como en sus distintos efectos sobre el DWB. En este sentido, PLN y empowerment podrían comportarse como extremos de un continuo en donde su nivel óptimo podría determinarse según el punto de equilibrio que lograra minimizar el nivel de ocurrencia del DWB. De esta forma, la dirección organizativa podría eventualmente contar con una herramienta contra el DWB en base a los niveles de PLNempowerment detectados en cada momento en la organización.

\section{Extended Summary}

\section{Introduction}

Workplace deviance labeled by Robinson and Bennett (1995:556) as deviant workplace behaviors (DWB), are “... any voluntary behavior that violate significant organizational norms and, in so doing, threaten the well-being of an organization, its members, or both." Examples of DWBs would include coming in late to work without giving prior notice and putting little effort into work. One study (Coffin, 2003 ) indicated that $75 \%$ of employees have taken property from their employers at least once. Other survey found that $42 \%$ of women reported being harassed at work (Gruber, 1990).

Given the above scenario, an increasingly amount of research on DWBs has centered on identifying the causes of such misbehavior in organizations. Alienation (from the Latin, alienati, alienare: 'take away', 'remove', 'or cause a separation to occur') has its origin in the writings of the German philosopher Karl Marx, and results from a contradiction between the nature of the work role and human nature. Work alienation (WA) - using the alienation concept as it was studied by Melvin Seeman (1971) - occurs in situations where the individual has lost control over the product and process of his labor, and hence the capac- ity to express herself or himself at work. This study suggests a relationship between WA and DWBs. As far as we know, work alienation (AL) as an antecedent of deviant behavior at work (DWB) has not been studied empirically within organizations settings. Address this gap in the literature is the focus of this work.

\section{Theoretical Background}

A group of terms associated with deviance has evolved. These actions are also defined as anti-social behavior (Robinson \& O'Leary-Kelly, 1998), organizational misbehavior (Vardi \& Wiener, 1996), noncomplaint behavior (Puffer, 1987), workplace deviance (Robinson \& Greenberg, 1998) and dysfunctional work behavior (Griffin, O'Leary-Kelly \& Collins, 1998), just to name a few. The definitions of these actions also vary. Gruys and Sackett (2003:30) labeled counterproductive workplace behaviors (CWB), and define them as "any intentional behavior on the part of an organizational member viewed by the organization as contrary to their legitimate interests." Robinson and Bennett (1995) labeled deviant workplace behaviors (DWB), and described 
them as any voluntary behavior that violate significant organizational norms and, in so doing, threaten the well-being of an organization, its members, or both. Their construct forms part of the basis in this paper. In a later study, Bennett and Robinson (2000) divided DWBs into two types: behavior that is directly harmful to the organization as a whole (DWBOs), and behavior that is more concerned with harming individuals within the organization (DWBIs). Examples of DWBOs would include coming in late to work without giving prior notice and putting little effort into work; whereas gossiping and blaming about coworkers would be examples of DWBIs.

Seeman (1971) provides the more contemporary interpretation of WA. In his initial article (1959), he attempted to clarify the meaning of the concept by identifying five dimensions of WA: powerlessness (i.e. lack of job autonomy by reason of which employees have limited freedom to exert control over work activities), meaninglessness (i.e. the inability to comprehend the relationship of one's contributions to a larger purpose), normlessness and isolation (i.e. when norms or codes of conduct do not effectively guide behavior toward personal goals), and self-estrangement (i.e. when work becomes a path for satisfying merely extrinsic needs rather than a means for expressing one's potential). Shepard (1973) defined alienation as awareness of the discrepancy between one's own inclinations and the demands of reality (see, also, Lee, 2005; Muntaner et al., 2006).

In predicting work behaviors, researchers usually propose a social exchange explanation (Blau, 1964) where employees may exhibit positive or negative behaviors as a response to the treatment received from their organizations. The use of the social exchange approach perspective would imply that to the extent employees feel alienate from their organization, the latter is unable to satisfy several employee needs, desires and preferences (Hirschfeld \& Feild (2000). As a result, WA would elicit negative sentiments (p.ej., lack of commitment, detachment, low self-esteem and dissatisfaction in general) which, in turn, would lead them to reciprocate with increased DWBs. to retaliate against the organization and behaviors toward the organization. Akroyd and Thompson (2000) argue in this direction when considering the DWB as a way in which alienated employees try to recuperate the lost control over the production process of their organization. Thus, by using Mottaz's (1981) original 21-item scale to assess felt alienation, which only includes dimensions of Powerlessness, Meaninglessness, and SelfEstrangement, we predict influences of the three WA dimensions of WA (powerlessness, meaninglessness, and self-estrangement) on the three types of DWB studied, i.e., directed at their organization (DWBO), co-workers (DWBIC), and students (DWBIS) - or clients (see Figure 1).

\section{Method}

\section{Sample Characteristics}

The questionnaires were distributed personally to all the teachers in a district high school in Gran Canaria (Spain) at the start of 2006. The response rate was $63.5 \%$ (99 out of 156). The sample comprised 53\% males and $47 \%$ females and, while $3 \%$ were 30 years old or younger, $55 \%$ were older than 40 . With regard to tenure, $36 \%$ of those responding had less than 10 years of experience, $50 \%$ had 10 to 20 years of experience, and $14 \%$ had over 20 years of experience working for the high school. Additionally, the sample descriptive structures are quite similar to the mentioned population data. Effectively, in the universe $53 \%$ of the teachers have 10 to 20 years of tenure, while $14 \%$ have over 20 years. By age, $3 \%$ were 30 years old or younger and $66 \%$ were older than 40 . By gender, $50 \%$ were male and $50 \%$ female.

\section{Measures}

All items of this study were scored on a 7-point scale ranging from (1) Strongly Disagree to (7) Strongly Agree-in DWB from (1) Never to (7) Constantly. Several items were negatively worded and thus were reverse reordered. Reliability was established by means of Cronbach's alpha, and the values of which are shown on the main diagonal of the correlations table (Table 1). All scales items are shown in the Appendix I.

Work Alienation. We used Mottaz's (1981) original 21-item scale to assess felt alienation, which includes dimensions of Powerlessness, Meaninglessness, and Self-Estrangement (7-item each one). All items of this study were scored on a 7-point scale. Alphas were calculated after some items, as presented in Appendix I, were rejected.

$D W B s$. The 10-item scale used to assess DWBs was a smaller version of the 18 -items proposed by Bennett and Robinson (2000). Indeed, certain items related to xenophobic behavior, or religiously discriminatory, among others, came not consider in our context sample. Therefore, eight were rejected. 3 items were added to measure DWBIA directed toward the student. The latter were constructed by the authors following the same DWBI pattern. The 13 resulting items then aim three-dimensionality (DWBO, DWBIC, DWBIA).

We then performed confirmatory factor analysis (CFA) for all variables to ensure that they were separate constructs by using structural equation modeling (SEM). Overall, fit of the model was assessed using multiple fit indexes. Results of CFA, shown in Appendix I, suggest that the variables are indeed distinct constructs. Next, after the final data were factor- 
ing analyzed (CFA), several items were dropped since they were loaded insufficiently (see Appendix I). Remaining items were averaged to work out correlations.

\section{Procedure}

We performed confirmatory factor analysis (CFA) for all variables to ensure that they were separate constructs by using structural equation modeling (SEM). Overall, fit of the model was assessed using multiple fit indexes. Results of CFA, shown in Appendix I, suggest that the variables are indeed distinct constructs. Next, after the final data were factoring analyzed (CFA), several items were dropped since they were loaded insufficiently (see Appendix I). Remaining items were averaged to work out correlations. Higherorder factor model were also calculated. As presented in Appendix II, the effects of WA on DWB were not significant $(B=-.04 ; p \mathrm{~ns})$.

\section{Results}

The model provided an excellent fit and therefore we accept their relationships. As such, the Chi-square was found to be scarcely significant $\left(\chi^{2}[3,99]=\right.$ $8,387, p=.039)$, the GFI was .97 ; the CFI was .94; the $\mathrm{NFI}=.92$, and RMR was .04 . Then, we analyzed each of the paths obtained (Figure 2). Thus, the results seem to support, and therefore allow us to accept Hypotheses $2 \mathrm{a}$ and $2 \mathrm{~b}$, as the meaninglessness significantly increased DWBO $(B=.22, p<.05)$ and DWBIC $(B=.27, p<.01)$. To our surprise, the link from powerlessness to DWBO $(B=-.21, p<.05)$, DWBIC $(B=-.29$, $p<.01)$ and DWBIA $(B=-.29, p<.01)$ was significant, but in the opposite direction than expected (Figure 2), and so Hypotheses 1a, $1 \mathrm{~b}$ and $1 \mathrm{c}$ do not receive support. Other relationships showed no significance, so we reject Hypotheses 3a, 3b, 2c and 3c.

\section{Discussion}

The objective of this research was to analyze the impact of WA on DWB. This is a relationship that, despite the profusion of studies on the alienation, has received limited attention in organizational settings. The results provide that, although unexpected, may offer important implications and offer to organizational managers of a range of options in their aim to combat DWB. These aims, combined with our results, we propose the conclusions and implications that we undertake in the lines below.

Perhaps mainly for its impact on organizational image, especially since the early 80 's, the particularities of the context in which the labor activity takes place have become increasingly important. Indeed, safety and hygiene at work, corporate policies to ensure equality of opportunity, or the detection of abusive behaviors within organizations have broad legal and economic implications. However, despite the abundant literature on WA and its significant impact of DWB on organizational effectiveness, the work of professionals in HR management to identify and mitigate situations, to develop methods or systems to deter dysfunctional work, have repeatedly ignored the perspective of the impact of WA on DWB provided in this study.

Therefore, a better knowledge of the relationship between WA and DWB can be helpful for HR managers and organizational management in general, to choose best tools and actions to minimize the occurrence of DWB and its harmful effects on the organization. The results of our study would support the use of communication strategies to raise awareness about the importance of employees about of their work, tasks, procedures and duties, and how they fit with the organization conduct, principles and values in order to avoid feelings of meaningless. Such actions, not only would have positive effect on the motivation of workers by reducing WA, but also would reduce harmful behaviors (DWB) would be expected by WA against the organization and coworkers. However, the results of our analysis suggest that efforts in decreasing DWB by avoiding feelings of powerlessness will not, at least, effective. In our mind, this would not imply that a policy of empowerment enhancing could come to trigger DWB. Rather, although not supported by the results of the study, a policy to eradicate feelings of powerlessness (if it is clearly assumed by authorities close to the employee) should also act positively in discouraging DWB.

Finally, we should also indicate that the study has limitations. First, the study could suffer from monomethod/source bias. Second, the high school that supplied the sample has certain job conditions that are often inherent to the peculiarities of workers in the public sector. For example, it might be debatable whether the studied variables in our school context would also be the same among employees in the private sector, which could present a different picture.

\section{Referencias}

Ackroyd, S. y Thompson, P. (2000). Organizational misbehaviour. Thousand Oaks, CA: Sage.

Bennett, R.J. y Robinson, S.L. (2000). Development of a measure of workplace deviance, Journal of Applied Psychology, 85, 349-360.

Bensimon, H.F. (1994). Violence in the workplace. Training and Development Journal, January, 28, 27-32.

Blau, P.M. (1964). Exchange and power in social life. New York: John Wiley. 
Blauner, R. (1964). Alienation and freedom: The factory worker and his industry. Chicago, Il: University of Chicago Press.

Case, J. (2000). Employee theft: The profit killer. Del Mar, CA: John Case \& Associates.

Coffin, B. (2003). Breaking the silence on white collar crime. Risk Management, 50, 8 .

Dean, D.G. (1961). Alienation: Its meaning and measurement. American Sociological Review, 26, 753-758.

Durkheim, E. [1893 (1984)]. The Division of labor in society. New York: Free Press.

Durkheim, E. [1897 (1951)]. Suicide: A study in sociology. New York: Free Press.

Foa, U.G. y Foa, E.B. (1975). Resource theory of social exchange. Morristown, NJ: General Learning Press.

Fromm, E. (1955). The sane society. New York: Rinehart.

Gouldner, A.W. (1960). The norm of reciprocity. American Sociological Review, 25, 161-178.

Griffin, R.W., O’Leary-Kelly, A. y Collins, J. (1998). Dysfunctional work behaviors in organizations. En C. L. Cooper y D. M. Rousseau (Eds), Trends in organizational behavior, Vol. 4 (pp. 65-82). Chichester: John Wiley \& Sons.

Gruber, J.E. (1990). Methodological problems and policy implications in sexual harassment research. Population Research \& Policy Review, 9, 235-254.

Gruys, M.L. y Sackett, P.R. (2003). Investigating the dimensionality of counterproductive work behavior. International Journal of Selection and Assessment, 11, 30-42.

Hirschfeld, R. R. y Feild, H. S. (2000). Work centrality and work alienation: Distinct aspects of a general commitment to work. Journal of Organizational Behavior, 21, $879 ? 890$.

Horowitz, I.L. (1966). On alienation and the social order. Philosophy and Phenomenological Research, 27, 230-237.

Johnson, F. (1973). Alienation: concept, term, and meanings. New York: Seminar Press Inc.

Jöreskog, K.G. y Sörbom, D. (1986). Lisrel IV: Analysis of linear structural relationship by maximum likelihood, instrumental variables, and least squares methods, $\left(4^{\text {th }}\right.$ ed,), Moorseville, IN: Scientific Software.

Kirsch, B.A. y Lengermann, J.J. (1971). An empirical test of Robert Blauner's ideas on alienation in work as applied to different type jobs in a white-collar setting. Sociology and Social Research, 56, 180-94.

Kohn, M.L. (1976). Occupational structure and alienation. American Journal of Sociology, 82, 111-30.

Korman, A. K., Wittig-Berman, U. y Lang, D. (1981). Career success and personal failure: Alienation in professionals and managers. Academy of Management Journal, 24, 342-360

Maslach, C. y Jackson, S.E. (1980). The measurement of experienced burnout. Journal of Occupational Behavior, 2, 99-113

Maslach, C. y Jackson, S. E. (1984). Burnout in organization- al settings. En S. Oskamp (Ed.), Applied social psychology manual (Vol. 5, pp. 133-154). Beverly Hills, CA: Sage.

Marx, K. (1844). Economic and Philosophical Manuscripts. Progress Publishers, Moscow 1959.

Molist, M. (2005). Empresas estadounidenses pierden miles de datos. VSantivirus, 1817, 1-3. http://www.vsantivirus. com/mm-28-06-05.htm

Mottaz, C.J. (1981). Some determinants of work alienation. The Sociological Quarterly, 22, 515-529.

Puffer, S.M. (1987). Pro-social behavior, non compliant behavior, and work performance among commission sales people. Journal of Applied Psychology, 72, 615-621.

Robinson, S.L. (2008). Dysfunctional workplace behavior. En: J. Barling y C.L. Cooper, (Eds.), The Sage handbook of organizational behavior, (pp. 141-159). Thousand Oaks, CA: Sage.

Robinson, S.L. y Bennett, R.J. (1995). "A typology of deviant workplace behaviors: A multidimensional scaling study", Academy of Management Journal, 38, 555-72.

Robinson, S.L. y Greenberg, J. (1998). "Employees behaving badly: dimensions, determinants, and dilemmas in the study of workplace deviance". En Cooper, C.L., Rousseau, D.M. (Eds), Trends in Organizational Behavior (Vol. 5, pp. 1-30), New York, NY: Wiley.

Robinson, S.L. y O'Leary-Kelly, A.M. (1998). Monkey see, monkey do: The influence of work groups on the antisocial behavior of employees. Academy of Management Journal, 41, 658-672.

Rosner, M. y Putterman, L. (1991). Factors behind the supply and demand for less alienating work, and some international illustrations. Journal of Economic Studies, 18, 18-41.

Sarros, C.J. (2002). Work Alienation and organizational leadership. British Journal of Management, 13, 285-304.

Schat A. y Kelloway, E.K. (2005). Workplace violence. En: J. Barling, E.K. Kelloway y M. Frone (Eds.), Handbook of workplace stress (pp. 189-218). Thousand Oaks, CA: Sage.

Seeman, M. (1959). On the meaning of alienation. American Sociological Review. 24, 783-791.

Seeman, M. (1967). On the personal consequences of alienation in work. American Sociological Review, 32, 273-84.

Shepard, J.M. (1973). "Technology, division of labor and alienation”, Pacific Sociological Review, 16, 61-88.

Shepard, J. (1977). Technology, alienation, and job satisfaction. Annual Review of Sociology, 3, 1-21.

Simpson, I. H. (1999). Historical patterns of workplace organization: From mechanical to electronic control and beyond. Current Sociology, 47, 47-75.

Vardi, Y. y Weiner, Y. (1996). Misbehavior in organizations: A motivational framework. Organizational Science, 7 , 151-165.

Williams, L.J. y Hazer, J.T. (1986). Antecedents and consequences of satisfaction and commitment in turnover models: A reanalysis using latent variable structural equation methods, Journal of Applied Psychology, 71, 219-231.

Manuscrito Recibido: 21/01/2010

Revisión Recibida: 05/04/2010

Aceptado: 05/03/2010 
Apéndice I. Análisis factorial confirmatorio (AFC) de las variables del estudio

\section{Medidas}

\section{Powerlessness}

X1 Dispongo de un amplio margen de libertad para desarrollar mi trabajo como crea conveniente $(R)$

$\mathrm{X} 2$ Tengo la oportunidad de aplicar mis propios criterios en el desempeño de mi trabajo $(R)$

$\mathrm{X}$ - Tengo muy poco control sobre cómo tengo que realizar mis tareas diarias $(*)$

$\mathrm{X}$ - La mayoría de las decisiones en mi trabajo las tomo sin tener que consultar a la directiva $(*)$

X- Me resulta difícil hacer cambios en la forma en que se establece cómo debo hacer mi trabajo $\left(^{*}\right)$

$\mathrm{X}-\quad$ Lo que tengo que hacer cada día está ya totalmente fijado de antemano $(*)$

$\mathrm{X} 3$ Yo tomo mis propias decisiones sobre los asuntos que surgen en mi trabajo $(R)$

\section{Meaninglessness}

X4 Mi papel en este centro contribuye mucho a su buen funcionamiento $(R)$

$\mathrm{X}-\quad$ A veces no estoy seguro de comprender la finalidad real de mi labor aquí (*)

X5 En verdad mi trabajo en el centro me parece que es importante y valioso $(R)$

$\mathrm{X}$ - A menudo me pregunto para qué sirve lo que estoy haciendo en mi trabajo $(*)$

$\mathrm{X}$ - Muchas veces siento que mi trabajo poco aporta a todo lo que aquí se hace $(*)$

X6 Consigo ver cómo mi labor profesional encaja dentro del funcionamiento global del centro $(R)$

$\mathrm{X} 7$ Soy capaz de comprender cómo mi trabajo forma parte de una labor colectiva del centro $(R)$

\section{Self-estrangement}

$X$ - No creo que sea precisamente una hazaña el desempeñar mi trabajo actual $(*)$

$\mathrm{X}$ - Actualmente mi sueldo es lo más importante de mi trabajo $(*)$

$\mathrm{X} 8 \quad$ Mi actual trabajo me hace sentirme realizado $(R)$

$\mathrm{X}-$ El trabajo que hago actualmente me impide hacer uso de mis reales habilidades y capacidades $(*)$

X9 Mi trabajo es una experiencia que me resulta altamente gratificante $(R)$

$X-$ No creo que sea precisamente una hazaña el desempeñar mi trabajo actual $(*)$

\section{DWBO}

$\mathrm{X}-$ Me he evadido en exceso fantaseando o pensando en cosas personales $(*)$

$\mathrm{X}-$ Sabiendo que iba a llegar tarde, no he avisado con antelación (*)

X11 He trabajado menos de lo que debiera

$\mathrm{X}-$ No he seguido, por diferentes motivos, las instrucciones de mi/s jefe/s $(*)$

$\mathrm{X} 12$ He puesto poco esfuerzo en mis tareas

X13 Me he tomado demasiado tiempo libre

\section{DWBIC}

$\mathrm{X}-\mathrm{Me}$ he pasado un poco discutiéndole a un compañero/a $(*)$

X14 No he hablado precisamente bien de algún compañero/a

X15 Me he pasado un poco bromeando con algún compañero/a

X16 He tratado mal a algún compañero/a

\section{DWBIA}

X17 Me he pasado un poco regañando a un alumno/a

$\mathrm{Cmin}=297.131 d f=215 p<0.001 \mathrm{Cmin} / \mathrm{df}=1.382 \mathrm{CFI}=0.91 \mathrm{NFI}=0.75 \mathrm{IFI}=0.91 \mathrm{RMSEA}=0.062 \mathrm{TLI}=0.88 \mathrm{PNFI}=0.58 .(*)$ Antes del cálculo de los índices del modelo eliminamos estos ítems dado que su carga factorial resultó ser inferior a 0.60 . $(R)$ Items formulados inversamente y recodificados antes de los análisis. 
Apéndice II. Modelo de Orden Superior de la Alienación, en sus tres Dimensiones y en las tres Dimensiones de las Conductas Desviadas en el Trabajo (DWB)

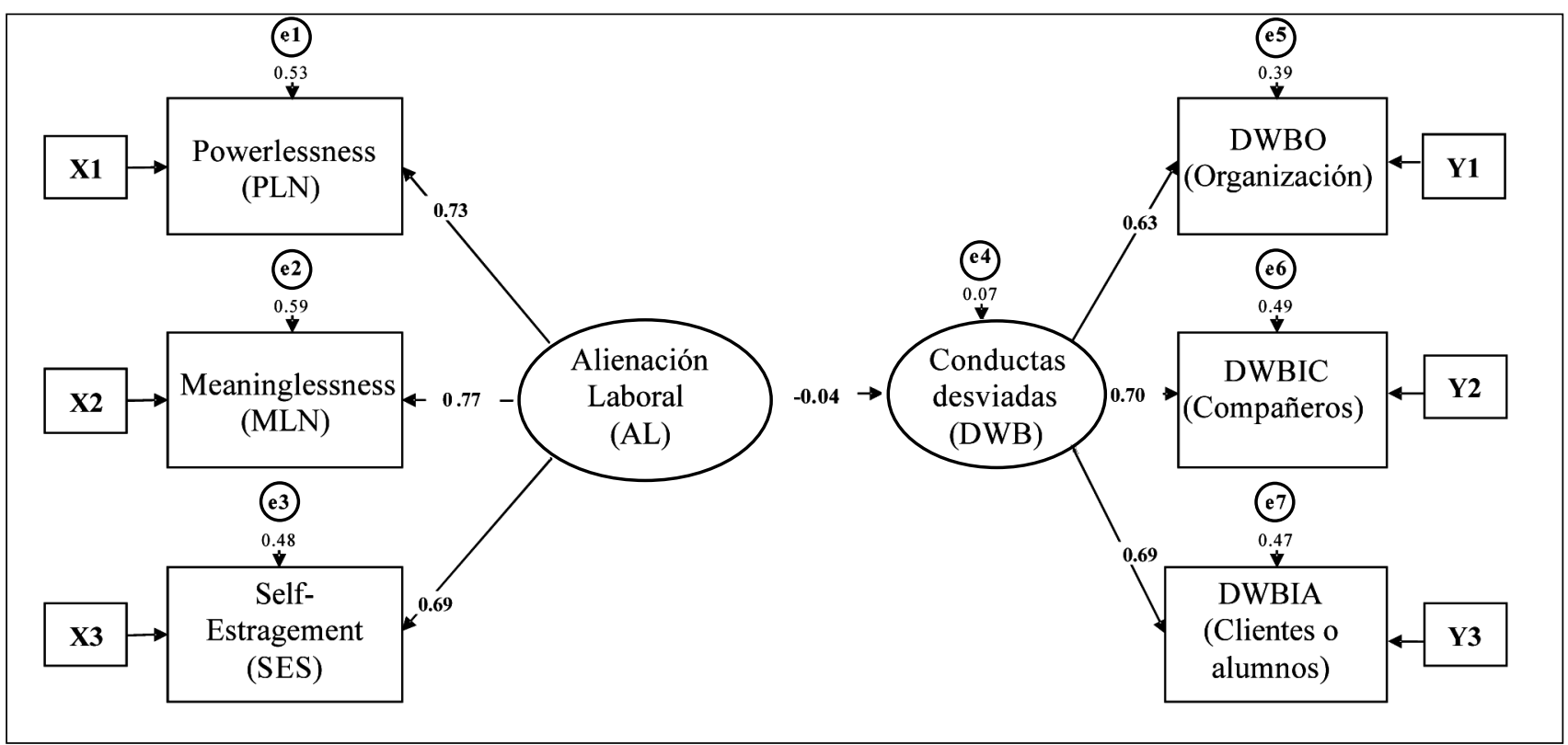

$(\chi 2[8,99]=13.060 ; \mathrm{p}=.110), \mathrm{GFI}=.99 ; \mathrm{CFI}=.96 ; \mathrm{TLI}=.93 ; \mathrm{NFI}=.91 ; \mathrm{RMR}=.03$. 